\title{
Impact of Meditation on Morality, Self-Control and Social Warmth of IT Professionals
}

\author{
Dr.Ramyashilpa.D.Nayak \\ Lecturer Department of Behavioural Science USM-KLE International Medical Programme \\ Belgaum Karnataka
}

\begin{abstract}
The present study is an extensive research of the impact of sridharanandji's Atma Vidya Parishilana Yoga on psychological personality variables before and after meditation as an intervention to software professionals. To face the modern day stress filled anxiety flooded world of IT [Information Technology] it is difficult for a professionals to lead a stress free life lacking personality traits like Morality, Self-control and Social Warmth. And these traits can be improved through daily practice of meditation. Thus the purpose of this research was to see effect of Sridharanandji's meditation on personality. Some of the personality dimensions such as morality, self-control and social warmth were measured through Multi-Dimensional Assessment of Personality MAP FORM-A before introducing them to Sridharanandji's technique of meditation. Thereafter the open eye technique of Sridharanandji was given to software professionals. The software professionals were psychologically prepared to practice meditation regularly at early morning hours. Finally, after three months practice of meditation personality dimensions [morality, self-control and social warmth] were measured again and significant changes were noticed as there was increase of morality, self-control and social warmth and overall improvement in personality after intervention program of open eye meditation of Sridharanandji.
\end{abstract}

Key Words: Meditation, Morality, Self-Control, Social Warmth.

\section{INTRODUCTION}

The IT field has brought new challenges to Indian Socio-economic and psychological structures. No economy will survive in the contemporary context unless it raises itself economically and psychologically to face ever changing new demands. India has chosen to march ahead to attain economic progress by following the foot prints of developed economy like the United States and as a result it has entered a big way into the software arena, and taken a quantum leap by providing employment to millions of technical graduates. By raising their income level exorbitantly combined with new problems of adjustments like lack of self-control, morality and social warmth which is an invariable by-product of a sudden economic change.

Common man is interested in the material attainment and maintenance of family which takes away major portion of his wakeful existence. He cannot devote long hours to decipher the intricacies of religiocultural moral principles. Therefore certain culture appropriate simple guidelines are to be laid down which are easy to practice and yield quick results in-terms of stress management and current adjustment to life's challenges.

Sat. Guru Sridharanandji dealt directly with the study of soul. He prescribed a very simple path, which is almost similar to Patanjali's Astanga Yoga. In Sat. Guru Sridharanandji's path certain culture appropriate, time conscious, diet conscious inter-personal sensitivity, law abiding are fundamentally required virtues. According to him breath is the sure media to suppress the modifications of mind or consciousness. All that is inhibited or present in the creation of both animate and inanimate is finally controlled, covered, modified and extinguished by the moving breath understanding it, saving our-self, benefiting from the knowledge of this; and never craving for the wealth of or belongings of others. Human being is born with a soul, the task of emancipation is to wipe out the entire images super imposed on the state of the being. All the experiences of the individual are recorded one over another creating a super structure of cognitive framework. Meditation will washout or substract one by one all impressions until the original fabric of supra-mental consciousness becomes clearly visible.

With passing of the time practitioner will acquire the technique of entering his true being in which all the sense organs will merge into the supreme power and thereby charged with the power of the supreme which helps them to lead the life dispassionately without being tossed by the contradictions of mundane life. Thus individual will attain indissoluble state of oneness which provides a supportive psychological setting for intellectual morality, self-control, social warmth and spiritual development of the individual. Naturally, he remains calm, collective and happy.

Sridharanandji's suggestion would be to restructure or reconstruct or don't anticipate or expect thoughts not to bother because thoughtlessness is not the ultimate goal. Thoughts remain active until final 
merger. If one don't expect that, thoughts do not bother, when they appear just observing them without trying to evaluate the thoughts. Unevaluated thoughts disappear and cannot reappear. In this way the appearance of thoughts backed by motivation operative on choice and context will gradually reach a point of no return. Through such practice self-control will significantly improve.

Sridahranandji stresses the need for waiting without any terminal point 'End' beings at point of the mundane. As Patanjali proclaims total surrender 'Ishwarasannidhana' or final surrender. Nobody knows where one ends. The ocean of peace dormant inside the abysmal depth of the being will charge the individual novel, self-controlled, well morel led, socially warm, dilative insightful, creative and he will notice by his 'Anthahkarana' or the third eye a delicate thread of all pervasive witness binding all the souls on the planet.

Psycho-physically a common man can drive lot of benefits from this method of meditation because a 15-20 minutes meditation during the early morning hours will redeem the individual of all toxins present in the body providing him a balanced state schism free state of consciousness.

Rosaen C, Benn R. (2006) studied the themes described by students resulting from meditation included the following: (1) an increasing state of restful alertness; (2) improvement in skills indicative of emotional intelligence (self-control, self-reflection/awareness, and flexibility in emotional response); and (3) improvement in academic performance. The state of restful alertness induced by meditation appeared central to facilitating growth in social-emotional capacities, academic performance, and flexibility in emotional response. The inner state of restful alertness provided students with greater capacity to expand their ways of looking inwardly at themselves and their relationships with others (emotional intelligence) as well as focusing their attention on controlling their behavior and keeping on task in school. Students described beneficial effects of TM: an increased state of restful alertness and greater capacity for self-reflection, self-control, and flexibility as well as improved academic performance. The state of restful alertness induced by meditation may facilitate the growth of social-emotional capacities necessary for regulating the emotional labiality and interpersonal stress of adolescence. Future empirical validation is needed to analyze systematically the impact of this practice on students' social-emotional and cognitive development and to determine whether its practice can serve as a protective function for helping students successfully meet the challenges of adolescence.

Statistical meta-analysis of all available research (42 independent outcomes) indicated that the effect of Maharishi's Transcendental Meditation Programme on increasing self-actualization is much greater than that of other forms of meditation or relaxation. Self-actualization refers to realizing more of one's inner potential, expressed in every area of life: integration and stability of personality, self-regard, emotional maturity, capacity for warm interpersonal relationships, and adaptive response to challenges.

Subjects practicing the Transcendental Meditation Programme, measured once prior to beginning the technique and again two months later, showed significant positive improvements in personality traits, including spontaneity, self-regard, and self-acceptance, when compared to a matched control group.

Those practicing the Transcendental Meditation Programme, and those practicing the TM-Sidhi Programme, showed higher - more principled-levels of moral reasoning on a standardized test in comparison to those who had not yet learnt the Transcendental Meditation Programme.

The present study examined whether it is possible to improve personality qualities morality, selfcontrol and social warmth with meditation practice. It attempts to investigate whether meditation helps in improving morality, self-control and social warmth. I tried to see whether meditation practice has any impact on personality change. Also, it explored the degree of congruence of personality both before and after the practice of meditation.

Aim:

\section{METHODOLOGY}

To develop the consciousness about the significance of sustaining a good personality and adopt certain personality characteristics such a self-control, morality and social warmth through the practice of open eye meditation technique of Sat.Guru. Sridahranandji

\section{Purpose:}

To enhance and strengthen the personality among the IT professionals and bring about strong characteristic changes in self-control, morality and social warmth through the practice of unique open eye Meditation Technique of Sat.Guru. Sridahranandji

\section{Objectives:}

- To find out the level of self-control, morality and social warmth before and after the practice of open eye meditation technique. 
- To see the impact of meditation on personality characteristics such as self-control, morality and social warmth.

\section{Hypothesis:}

- Practice of meditation has significant effect on improving personality characteristics such as selfcontrol, morality and social warmth.

\section{Sample:}

The study was carried out on one group of software professionals who do not practice any kind of yoga or meditation. The group consisted of 108 subjects ( 54 men and 54 female) in the age range of (24-45 years). They were randomly selected from the Bangalore IT companies of Karnataka state.

All the participants were administered personality test [Multi- Dimensional Assessment of Personality MAP Form-A] before they were thought to practice Sridhranandji's meditation technique. Then after the participants were introduced to meditation and they practiced regularly for 15-20 minutes. After the practice of meditation for three months again the same personality test was carried out.

\section{Assessment Tool}

MAP- From-A [Multi-Dimensional Assessment of Personality] has 147 items, which cover twenty normal personality dimensions. For the present study three of the dimensions morality, self-control and social warmth ware chosen and each dimension consist of 7 items. Each item in the questionnaire has three choices from which the subject may select one answer.

\section{Results and Discussion:}

The obtained 'p' value for morality is $.000(\mathrm{p}<001)$, self-control is $.000(\mathrm{p}<001)$ and for social warmth it is .003 $(\mathrm{p}<001)$ which show highly significant correlation. The correlation scores are positive and statistically significant after the practice of meditation. In other words in this study participants after meditation experienced greater congruence in personality than before the practice of meditation. Laurence Nixon researched on the Psychological Role of Meditation in Personality Change From the Perspective of the Theory of Positive Disintegration and in his study he says that the preconditions for meditation as well as the effects of the practice on the development of personality from the perspective of the theory of positive disintegration. According to him the effect of meditation is to facilitate the emergence of developmental dynamisms needed for personality growth.

Results indicate that after the meditation practice for three months significant differences were found in the software professionals. They scored high on morality, self-control and social warmth. Thus it may be said that practice of meditation appears to have some significant influence on morality, self-control and social warmth dimensions of personality. This may be further verified with larger samples and with long term practitioners of meditation before the relationship is confirmed. In the resent study it is also observed that software professionals after practicing meditation showed greater congruence of morality, self-control and social warmth than before the experience of meditation.

\section{CONCLUSION}

In conclusion the study result reveals the personality characteristics improve with the practice of meditation. The practice of meditation found fruitful in developing self-control, morality and social warmth which helps the IT professionals at busy work life and managing a good balance with the personality characteristics. It has been concluded that though the practice of meditation it improves and enhances the personality traits on the whole and helps the professionals to lead a well-balanced life.

\section{Significance of the Study}

As we see the research results clearly reveal that how meditation brings about changes in the morality, selfcontrol and social-warmth it important for us to carry out similar researches so that a broad awareness can be created regarding the significance of meditation and its impact of personality. To say in broad sense it's not only the IT professionals who face problems with personality but there are many other laymen who have an imbalanced personality. Hence it is important for us to create a cognizance regarding meditation and encourage more and more people to practice meditation and not only to improve personality but also to achieve physiological balance and free from stress and anxiety and many more other problems. 


\section{References}

[1]. Dabrowski, K. Personality-Shaping through Positive Disintegration. London: J. \& A. Churchill, 1967.

[2]. Rosaen C, Benn R (2006) The experience of transcendental meditation in middle school students: a qualitative report. Department of Family Medicine, University of Michigan Integrative Medicine, Ann Arbor, MI 48104, USA. U.S. National Library of Medicine National Institutes of Health. PMID: 16979106 [PubMed - indexed for MEDLINE]

[3]. Maharishi (1991) Transcendental Meditation Programme on increasing self-actualization. Journal of Social Behavior and Personality 6(5): 189-248

[4]. Transcendental Meditation Programme, (1972) Journal of Counseling Psychology 20 184-187

[5]. Transcendental Meditation Programme, (1973) Journal of Counseling Psychology 20 565-566

[6]. Transcendental Meditation Programme, (1974) Journal of Counseling Psychology Perceptual and Motor Skills 39: 623-628.

[7]. The Transcendental Meditation and TM-Sidhi Programme' (1982). Paper presented at the International Symposium on Moral Education, University of Fribourg, Switzerland, Scientific Research on Maharishi's Transcendental Meditation and TM-Sidhi Programme - Collected Papers, Volume 3 (1990e): 2034-2037

[8]. M/S PSY-COM Services (1993, 1996). Handbook for MAP (Multi- Dimensional Assessment of Personality). Copyright B-4, 80/2, S’ Jung Endave, New Delhi-110 029 (India) 